\title{
Enhancing intentions to attend cervical cancer screening with a stage-matched intervention
}

\author{
Aleksandra Luszczynskal,2*, Gabriela Goc ${ }^{3}$, Urte Scholz ${ }^{4}$, \\ Monika Kowalska ${ }^{5}$ and Nina Knoll ${ }^{6}$ \\ 'Warsaw School of Social Sciences and Humanities, Poland \\ ${ }^{2}$ Trauma, Health, and Hazards Center, University of Colorado \\ at Colorado Springs, USA \\ ${ }^{3}$ Warsaw University, Poland \\ ${ }^{4}$ University of Zurich, Switzerland \\ ${ }^{5}$ Cardinal Stefan Wyszynski University, Warsaw, Poland \\ ${ }^{6}$ Charite - Universitätsmedizin Berlin, Germany
}

\begin{abstract}
Objective. The study evaluated the effects of a pros enhancing intervention on intention to uptake cervical cancer screening. It was hypothesized that the pros enhancement session (compared to an education session) would affect intentions of preintentional women, whereas it was expected to have negligible effects among women in intentional and actional stages of the health action process approach (HAPA). Thus, we tested the HAPA using stage-matched and stage-mismatched interventions. Further, a change in decisional balance was assumed to mediate the relationship between the group assignment and intention, with age acting as the moderator.

Design and methods. Respondents (1,436 women, aged 18-60) were randomly assigned to either the intervention or control condition and filled out questionnaires before and directly after the manipulation (in one web-based session).

Results. Direct effects of the group assignment were observed only among preintentional women. Across the stages, however, change in decisional balance mediated the effects of the group assignment on the intention to uptake screening. Further, among participants in the preintentional stage, this mediation became significant only for women aged 35 or older.

Conclusions. Although direct effects are in line with stage assumptions of the HAPA, meditational analysis among pre- and post-intentional women indicated that similar processes accounted for post-manipulation intention. Future research testing stage models should account for the mediating processes, which explain the effects of the intervention.
\end{abstract}

\footnotetext{
* Correspondence should be addressed to Dr Aleksandra Luszczynska, Trauma, Health, and Hazards Center, University of Colorado at Colorado Springs, 1420 Austin Bluffs Parkway, PO Box 7150, Colorado Springs, CO 80933-7I 50, USA (e-mail: aluszczy@uccs.edu).
} 
The benefits of cervical cancer screening include the diagnosis and treatment of disease in preinvasive stages, as well as a shift in incidence of invasive disease from advanced to early (treatable) stages (Hakama, Coleman, Alexe, \& Auvien, 2008). Therefore, regular screening affects reduction of cancer incidence (Hakama et al., 2008). Although the evaluation of the effectiveness of the screening programmes varies in terms of relative risk reduction, specialists agree that the cytological smears have convincingly shown to reduce the mortality rates (Hakama et al., 2008). It is usually recommended to start cervical cancer screening between age of 18 and 30 (Hakama et al., 2008).

Age is a strong predictor of cervical cancer screening uptake with younger women (below 30 or 35 years old) underutilizing screening programmes (Byrd, Peterson, Chavez, \& Heckert, 2004; Duffett-Leger, Letorneau, \& Croll, 2008; Langille \& Rigby, 2006; Tacken et al., 2007). Recent studies on the prevalence of human papilloma virus (HPV) infection as an antecedent of cervical cancer indicate that a large proportion of young women in the early phase of their sexual life carry an HPV-infection (De Vuyst, Clifford, Li, \& Franceschi, 2009). Cervical cancer may originate in a fraction of women with a persistent high-risk HPV-infection, usually within early post-menopausal years (Franco, Schlecht, \& Saslow, 2003). Such complexities in early risk-factor acquisition and associated but delayed disease onset may add to the underutilization of screening programmes for cervical cancer by younger women.

Stages of change for cervical cancer screening are predicted by age (Hogenmiller et al., 2007; for the description of stages, see Rakowski et al., 1996). Age moderates the effectiveness of interventions promoting cervical cancer screening, with older women benefiting more from the interventions addressing risks perceptions (Fernandez-Esquer, Espinoza, Torres, Ramirez, \& McAlister, 2003). Thus, research predicting cervical cancer screening participation and evaluating the effects of respective interventions should account for a moderating role of age.

\section{Beliefs and cervical cancer screening: Health action process approach, stages of change, and decisional balance}

According to the health action process approach (HAPA), a behaviour change intervention should account for stages of change, which are marked by past behaviour and intentions (Schwarzer, 2008). Individuals in earlier stages had never performed a particular screening behaviour and they would indicate that they neither intend nor plan to perform screening within 1-2 years, or they may just start considering initiating screening in next 1-2 years (Rakowski et al., 1996). This stage is referred to as preintentional (Schwarzer, 2008). Intentional stage is defined by a lack of past behaviour and an intention to uptake it soon. Finally, women who have engaged in screening behaviour at least once (within the recommended time-span) or have performed regular screening in the past (Rakowski et al., 1996), and intend to engage in screening behaviour in the future can be classified as actional individuals (Schwarzer, 2008). Although according to Rakowski et al. (1996) the cervical cancer screening time frame refers to 1-2 years, this has to be adjusted to the screening recommendations in a particular country.

Pros and cons are behaviour-related beliefs which can be found across theories of behaviour change (Schwarzer, 2008; Sutton, 2005). The perceived advantages (pros) and disadvantages (cons) of behaviour change form a decisional balance (Janis \& Mann, 1977). The HAPA indicates that perceived advantages and disadvantages should prompt individuals in preintentional stage (i.e., among individuals who have not developed 
strong intentions). The HAPA suggests that pros have a minor effect in more advanced stages, when intention is strong or if actions have already been initiated (Schwarzer, 2008). Indeed, interventions addressing pros may be of particular importance for women in early stages of change (Smith, Kloss, Kniele, \& Anderson, 2007). Research indicated that decisional balance is usually related to past behaviour and intention to engage in cervical cancer screening in future (Kelaher et al., 1999; Tung, Nguyen, \& Tran, 2008).

\section{Effects of stage-matched and stage-mismatched interventions on behaviour change}

Several studies indicated that pros are related to intention and screening behaviour across stages of change (Hou, Fernandez, \& Chen 2003; Kelaher et al., 1999; Rimer et al., 1999; Tung et al., 2008). Therefore, many interventions aiming at prompting intention or behaviour change recruited all women, regardless of their stage of change. Compared to a standard education session, discussing personal pros over phone (plus receiving printed materials about the pros and cons) resulted in similar Papanicolaou (pap) test attendance (61 vs. 70\%; Rimer et al., 1999). Among women aged 20-64 who either received the information on pros and cons of cervical cancer screening or education materials, those who participated in pros enhancement declared that they intend to perform pap test less frequently (79\%) than those who received standard care (88\%) (Adab et al., 2003). Rimer et al. (1999) found that providing printed materials on pros and cons had no effect on screening attendance, and even resulted in slightly less frequent screening behaviour (59\%) than reminding about the screening by a physician (61\%). Such results could be interpreted as indicative of low effectiveness of an intervention targeting pros, because participants across all stages of change are enrolled. Interventions which adjust the targeted cognitions to participants' stages of change can be called stage-matched interventions. By contrast, interventions enhancing cognitions which according to a theoretical model are of lower relevance in a particular stage may be called stagemismatched interventions. If the study aims at enhancing pros among all participants, the intervention would be stage-matched for those in preintentional stage, whereas it would be stage-mismatched for those in intentional and actional stages.

Stage models and research providing support for the notion of stages have been criticized. West (2005) suggested some flaws in the theoretical foundations of these models, whereas Sutton (2005) showed that the empirical evidence for the existence of stages of change is rather limited. Comparing effects of the stage-matched and stagemismatched interventions may provide the strongest evidence for the stages models (Sutton, 2005). This approach, however, was rarely applied.

Evidence supporting stages of the HAPA includes several non-experimental studies (Lippke, Ziegelmann, \& Schwarzer, 2005; Renner \& Schwarzer, 2005; Schuez, Sniehotta, Mallach, Wiedemann, \& Schwarzer, 2009). An experimental study (Lippke, Ziegelmann, \& Schwarzer, 2004) showed that a planning intervention had a stage-matched effect (i.e., was helpful for intenders), whereas participants from other stages did not benefit from forming plans. We found no study testing the assumptions of the HAPA, applying stage-matched interventions among preintentional individuals.

Besides testing if an intervention works, it is necessary to indicate how it actually works (Michie \& Abraham, 2004). Disclosing the underlying mechanisms may be easier if the intervention targets a specific belief and changes in this belief are found to mediate the relationship between the group assignment and the outcomes (Michie \& Abraham, 
2004). If this mediating effect would be specific for the subgroup in which stage and the intervention match each other, such result would provide strong support for a stage model.

\section{Aims of the study}

We investigated the relationships between the intervention addressing advantages to uptake cervical cancer screening, stages of change, and the post-intervention intention to engage in screening behaviour. First hypothesis assumed that compared to the control condition (education) the intervention would affect the intention among preintentional women (a stage-matched intervention), whereas no effect of the group assignment was expected among women in other stages of change (i.e., a stage-mismatched intervention). Second hypothesis assumed that among preintentional women the effects of the intervention on intention would be mediated by a change in decisional balance. As age constitutes a risk factor for low screening participation and moderates the effects of screening promotion programmes, it was tested if age moderates the effects of the pros enhancing intervention on intention.

\section{Method}

\section{Participants and procedures}

A total of 1,436 women agreed to take part and provided responses to all study sections. They were $18-60$ years old $(M=26.43, S D=7.89)$. Most of the respondents $(69 \%)$ were between 20 and 30 years; $9 \%$ were $18-20$ years old, $11 \%$ were $31-40$ years old, $7 \%$ were 41-50 years old, $4 \%$ were above 51 years old. None reported having had hysterectomy.

Data were collected on-line for 4 months in 2007. The study was advertised at three Polish websites dealing with women and health issues. A brief introduction was followed by the link to the website, where potential respondents were asked to provide an informed consent. They were informed about the study aims, anonymity, and that the study does not have to do with any commercial purposes and it is not sponsored by a pharmaceutical or medical technology company. Those who agreed to participate were linked to the survey website.

The study was conducted in one web-based session. Materials consisted of three sections: the initial (baseline) questionnaire, the control or the intervention group materials, and the post-test questionnaire. The pre-test started with a brief definition of cervical cancer screening, followed by questions about past behaviour, stage of change, intentions to live a healthy life, and a measure of perceived pros and cons of engaging in regular cervical cancer screening. Then respondents received a filler task dealing with a variety of beliefs referring to cervical cancer.

After finishing the initial section women were randomly assigned to the intervention $(N=712)$ or the control group $(N=724)$. The randomization was carried out using a random digit generator. No stratification was applied. The respondents were not aware of their group assignment. The control group participants received education materials including information about the aims and procedures involved in cervical cancer screening, the recommendations about the frequency of screening, need for testing across the age groups, and the relevance of screening even if the HPV inoculation was performed. 
Those assigned to the intervention group received the abbreviated education materials, followed by the intervention forms. First part of these forms was developed in an elicitation study, conducted in the month prior to the actual research among 30 young and middle-aged women ( $36 \%$ declared regular cervical cancer screening). Elicitation study participants were asked to provide responses to four open-ended questions referring to advantages and personal reasons for and against regular cervical cancer screening. Based on provided responses, we developed five brief vignettes discussing advantages of cervical cancer screening (suggesting that these advantages were indicated by other women participating in the study). The vignettes did not focus on increasing perceived risk of cervical cancer. Instead, the material stressed the following positive outcomes of screening behaviour: gaining control over one's own body and health and feeling pride in own efficient actions; helping to detect even minor changes in reproductive health; joining the community of women across the world who do similar things to take care of themselves and therefore protect their families; easy accessibility of the actual examination which can be done by a female nurse who can discuss related health issues with you; wider availability of more sensitive tests which can be obtained free of charge (via a national screening programme). The vignettes were followed by three open-ended questions where women were asked to list the advantages of cervical cancer screening which are the most relevant for themselves and which could motivate a woman friend or relative to uptake screening.

The intervention and control group materials were followed by a filler task dealing with other beliefs referring to the cervical cancer. The post-test included the assessment of pros, cons, and intention to attend cervical cancer screening. The participants were thanked and informed that a debriefing (or discussion of issues addressed in the study) is available by e-mail. Filling out all questionnaires and study materials (including pre- and post-test, control, or intervention group forms) took between 40 and $55 \mathrm{~min}$.

\section{Measures}

\section{Past behaviour}

Past bebaviour was measured at the baseline with two items, 'Within last three years (or within the period recommended by the doctor) I have took part in cervical cancer screening' and 'I have attended my cervical cancer screening within last 12 months'. Past behaviour measure referred to the 3-year period (and doctors' recommendations), as indicated in the cervical cancer screening guidelines in the country of the study. The response scales were ranging from 1 (definitely not) to 5 (definitely yes). The correlation between the items was .83 . Mean item responses ranged from 1 to $5(M=3.55, S D=$ 1.80).

\section{Stages of change}

Stages of change were evaluated at the baseline using the algorithm for cervical cancer screening (Rakowski et al., 1996) and stage algorithms for HAPA (Schwarzer, 2008), and divided into (1) preintentional, (2) intentional, and (3) actional groups. Participants were asked to indicate the item describing their current situation and beliefs: (1) no previous cervical cancer screening and either (a) no intention to get one within next 13 years or (b) considering a possibility to get one within next 1-3 years, (2) no previous cervical cancer screening but an intention to get one within next 30 days, (3) has had previous cervical cancer screening within last year (or in last 3 years, or in the period 
recommended by the doctor), and an intention to have another within next 12 months (or in the period recommended by the doctor). In general, the stage algorithm referred to the 3-year period (and doctors' recommendations), as indicated in the cervical cancer screening guidelines in Poland.

\section{Intention}

Intention at baseline referred to living a healthy life and was measured with three items, 'Within next months I intend to (a) invest more efforts to protect my health, (b) take more time and draw more attention to potential women health issues, (c) live a healthier life'. Responses were given on a five-point scale (ranging from definitely false to exactly true), with mean item response of $4.11(S D=0.84)$. Cronbach's alpha was .87 . To measure intention to engage in cervical cancer screening at the post-test participants were asked if they intend to uptake screening within next month (or a soon as recommended), then repeat screening behaviour 1 year later (or after a recommended time elapsed), and finally if they intend to repeat screening regularly during the 3-5 following years. This general question was followed by the items: 'I intend to engage in cervical cancer screening as soon as it is recommended (e.g., within next 30 days if I have not done it last year)', 'I intend to attend cervical cancer screening again at one year later (i.e., to repeat cervical cancer screening)', 'Within the following years I intend to take part in regular cervical cancer screening (e.g., on the annual basis or less/more frequently, if I have received different recommendations)'. Because some women may have done or intend to do their next screening on schedule, but they do not intend to continue in a long run (Rakowski et al., 1996), our measure was designed to capture intentions of uptaking screening soon, repeating it once, and repeating screening regularly over the following years. The responses were given on a five-point scale (definitely not to definitely yes). Mean item response ranged from 1 to 5, with the mean of 4.33 ( $S D=$ 0.91 ), Cronbach's alpha was .85.

\section{Pros and cons}

The measure of pros and cons consisted of 14 items presented in a random order at both pre- and post-test. The stem preceding the list of pros and cons was 'What would be your personal pros and cons of regular screening for cervical cancer'. The responses were given on a scale from 1 (definitely not) to 4 (exactly true). Pros included seven items derived from earlier studies (Rakowski et al., 1998; Rimer et al., 1999) and the elicitation study and included such examples as 'It would increase my sense of safety', 'I would be glad that I take care of my health', 'It would be good for me and my life partner'. Mean item response was $3.49(S D=0.58)$ at the baseline and $3.47(S D=0.65)$ at the post-intervention. Cronbach's alpha was .86 and .91 pre- and post-test. Cons were measured with seven items such as 'I would be afraid that I would get bad news', 'It would take quite some effort to organise the visit, collect the results, etc.', 'It would be an uncomfortable procedure'. The mean item responses were $2.19(S D=0.61)$ and $2.22(S D=0.68)$, at pre- and post-test. Cronbach's alpha was .71 and .80 at the pre- and post-test.

To calculate decisional balance mean scores obtained for cons were subtracted from pros mean scores at pre- and post-test (the higher the value the more positive the decisional balance). At baseline the balance ranged form -2.43 to +3.00 , with mean of $1.31(S D=0.92)$, whereas post-test balance ranged from -3.00 to $+3.00(M=1.25$, 
$S D=1.00$ ). Change in decisional balance was defined as baseline scores subtracted from post-test scores (higher values indicating a shift towards pros outweighing cons).

\section{Data analysis}

First hypothesis was tested using analysis of covariance. Moderated mediation procedures recommended by Preacher, Rucker, and Hayes (2007) were applied to test the second hypothesis. A moderated mediation model to predict post-manipulation intention was specified with covariates (baseline intention to live a healthy life, past behaviour), using the MODMEDC macro (Version 1.0; Model 5) by Preacher and Hayes (2004). The group assignment was the independent variable and change in decisional balance was the mediator. Age was the moderator of the effect of independent variable on the mediator and the effect of the mediator on the dependent variable. Centred variables were used (Aiken \& West, 1991), and groups were coded as -1 (the control condition) and +1 (the intervention). Missing data (below 1\%) were imputed using the expectation maximization algorithm (Enders, 2001).

\section{Results}

\section{Randomization check, manipulation check, and preliminary analyses}

Among the respondents, 52\% declared that they have done cervical cancer screening within the last 12 months and 59\% declared that they have done regular screening for cervical cancer within 3 years prior to the study. Among women aged 30 years old or less, the uptake of screening was $49 \%$ within last 12 months ( $65 \%$ among women older than 30 years old) and 54\% within 3 years prior to the study (83\% among women older than 30 years old). Differences between these two age groups were significant for screening in the last 12 months, $\chi^{2}(4,1436)=31.09, p<.001$, and last 36 months, $\chi^{2}(4,1436)=90.41, p<.001$. After applying the staging algorithm, $36 \%$ were classified as preintentional, $9 \%$ were in intentional stage, $55 \%$ were actional participants (mean intention: $4.18, S D=0.82$ ).

Participants assigned to the experimental and control groups did not differ in terms of past behaviour, $F(1,1435)=3.78$, ns, baseline cognitions such as intention to live a healthier life, $F(1,1435)=0.26$, ns, pros, $F(1,1435)=3.25$, ns, cons, $F(1,1435)=1.46$, ns, decisional balance, $F(1,1435)=2.85$, ns, and age $F(1,1435)=3.39$, ns.

To test if the intervention affected perceived pros and decisional balance repeated measures analysis of variance was performed. Regarding perceived pros, there was no significant effect of time, $F(1,1432)=1.71$, ns, no significant effect of time $\times$ stage interaction, $F(1,1432)=0.03$, ns, but there was a significant time $\times$ group assignment interaction, $F(1,1432)=11.20, p<.001, \eta^{2}=.01$. The interaction of time $\times$ stage $\times$ group assignment was not significant, $F(1,1432)=0.24$, ns. The intervention group participants indicated less pros at the pre-test $(M=3.51, S D=0.56)$ than at the post-test $(M=3.54$, $S D=0.61$, whereas the control group respondents indicated more pros at the pre-test $(M=3.47, S D=0.58)$ than at the post-test $(M=3.41, S D=0.67)$. Regarding decisional balance, there was a significant effect of time, $F(1,1432)=9.81, p<.01, \eta^{2}=.01$, no significant effect of time $\times$ stage interaction, $F(1,1432)=0.11$, ns, a significant time $\times$ group assignment interaction, $F(1,1432)=36.06, p<.001, \eta^{2}=.03$. The interaction of time, stage, and group assignment was not significant, $F(1,1432)=1.06$, ns. Women 
participating in the intervention reported lower decisional balance at the pre-test $(M=$ $1.36, S D=0.07)$ than at the post-test $(M=1.40, S D=0.97)$, whereas controls reported higher decisional balance at pre-test $(M=1.30, S D=0.92)$ than at the post-test $(M=$ $1.13, S D=1.01$ ).

Correlations between the study variables are displayed in Table 1. Older participants were more likely to have cervical cancer screening and reported stronger intentions to engage in screening behaviour in the future. Additionally, older participants perceived less cons at pre- and post-test and indicated more positive balance. Pros measured before and after experimental manipulation were strongly related. Significant associations were also found for the decisional balance measured at pre- and post-tests.

\section{The effects of the manipulation on intentions}

First hypothesis addressed the effect of the group assignment and stages of change on intention to engage in cervical cancer screening. Analysis of covariance was performed, as items with different wording were used to measure intention at pre- and posttest. We found a significant effect of the group assignment, $F(1,1429)=6.30, p<$ $.01, \eta^{2}=.01$, a significant effect of stages, $F(1,1429)=30.39, p<.001, \eta^{2}=.02$, and a significant stages $\times$ group assignment interaction, $F(1,1429)=36.98, p<.01$, $\eta^{2}=.01$. Preintentional individuals reported lower intentions in the control group than in the experimental group, $F(1,523)=9.49, p<.01, \eta^{2}=.02$, Cohen's $d=$ 0.23 (Figure 1). Women in more advanced stages reported similar intentions in both conditions $F(1,911)=1.27$, ns, Cohen's $d=0.10$ (Figure 1 ). Baseline intention to live a healthy life was a significant covariate $F(1,1429)=17.51, p<.001, \eta^{2}=.02$, together with past behaviour, $F(1,1429)=61.81, p<.001, \eta^{2}=.04$ and baseline decisional balance, $F(1,1429)=118.61, p<.001, \eta^{2}=.08$, whereas effects of age were not significant, $F(1,1429)=0.46$, ns.

\section{Moderated mediation analysis: The role of age and changes in decisional balance}

Second hypothesis assumed that the change in decisional balance should mediate the relationship between the group assignment and intention to uptake screening, particularly among preintentional women participating in the stage-matched intervention. It was also tested if age would moderate the effects of the intervention.

First set of analyses included data from women assigned to preintentional stage $(N=$ 524). The group assignment had an effect on intention to uptake screening ( $B=0.12$, $S E=0.05, p<.01)$. Change in decisional balance was predicted by the group assignment $(B=0.07, S E=0.02, p<.01)$, whereas covariates (intention to live a healthy life, past behaviour) were unrelated to the mediator. Age moderated the effect of the group assignment on the decisional balance change $(B=0.02, S E<0.01, p<.001)$. Controlled variables were associated with intention to uptake screening, with $B=0.10(S E=0.02$, $p<.001)$ for baseline intention to live a healthy life and $B=0.10(S E=0.01, p<.001)$ for past behaviour. Although the effect of the mediator (decisional balance change) on the dependent variable was not significant $(B=0.02, S E=0.08$, ns), the mediator by moderator interaction predicted intention at the post-test $(B=0.02, S E<0.01, p<$ $.01)$, with the conditional indirect effect of $0.13(S E=0.06, p<.05)$. The mediation became significant when the moderator (age) value was 34.34 or higher. Standardized 
Table I. Correlations between the study variables

\begin{tabular}{|c|c|c|c|c|c|c|c|c|c|c|}
\hline No. & Variable & 2 & 3 & 4 & 5 & 6 & 7 & 8 & 9 & 10 \\
\hline 1. & Intention to live a healthy life at baseline & .04 & $.31 * * *$ & $-.09 * *$ & $.26^{* * * * k}$ & $.30 * * *$ & $-.08 * *$ & $.24^{* k * k}$ & $.20^{* * * * * *}$ & -.01 \\
\hline 2. & Past behavior & & $.15^{* * * *}$ & $-.45 * *$ & $.40 * * * *$ & $.14^{* 1 * k}$ & $-.42^{* k * k}$ & $.37 * * *$ & $.54 * * *$ & $.29 * *$ \\
\hline 3. & Pros at baseline & & & -.18 \%10k & $.75^{* 1 * k}$ & $.84^{* 10 k}$ & $-.16 * 10 k$ & $.65 \%$ & $.34^{*+1 \times k}$ & -.03 \\
\hline 4. & Cons at baseline & & & & $-.78^{* k 1 k}$ & $-.16^{* 12 k}$ & .81 *kok & $-.65^{5 * * *}$ & $-.40 * 0 \%$ & $-.18^{* 10 *}$ \\
\hline 5. & Decisional balance at baseline & & & & & $.64^{* * 1 * k}$ & $-.64 * k$ & $.84 *$ 冰 & $.79 *$ *ok & $.10 * * *$ \\
\hline 6. & Pros at post-test & & & & & & $-.13^{* k 1 k}$ & $.74 * * *$ & $.33^{* * 1 \%}$ & -.02 \\
\hline 7. & Cons at post-test & & & & & & & $-.77 * 1 *$ & $-.36 * * *$ & $-.20^{* \text { *tok }}$ \\
\hline 8. & Decisional balance at post-test & & & & & & & & $.46 * 1 \%$ & $.12 * * *$ \\
\hline 9. & Intention to uptake cervical cancer screening (post-test) & & & & & & & & & $.15 * * *$ \\
\hline 10. & Age & & & & & & & & & \\
\hline
\end{tabular}

$* x p .01 ; * * * p<.001$. 


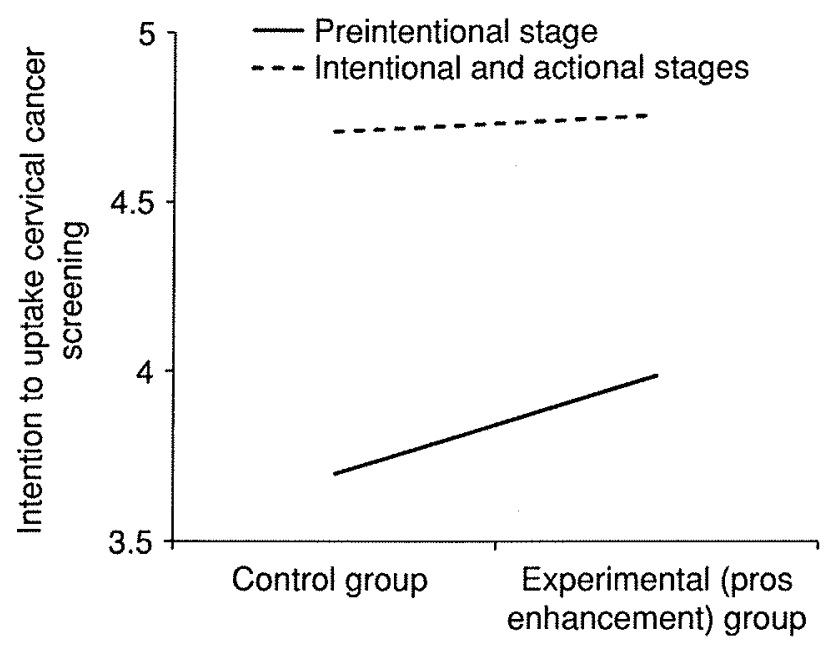

Figure I. Effects of pros enhancing intervention on intention to uptake cervical cancer screening among women from preintentional stage and those from more advanced (intentional and actional) stages.

solution for moderated mediation analysis for preintentional women is presented in Figure 2.

The moderated mediation analysis was repeated for the participants in intentional and actional stages, lumped together $(N=912)$. Change in decisional balance was predicted by the group assignment $(B=0.07, S E=0.01, p<.001)$, whereas covariates were unrelated to the mediator. Age did not moderate the effect of the group assignment on the decisional balance change, $B=0.01(S E=0.01$, ns). Intention was predicted by change in decisional balance $(B=0.07, S E=0.02, p<.05)$ and it was marginally related

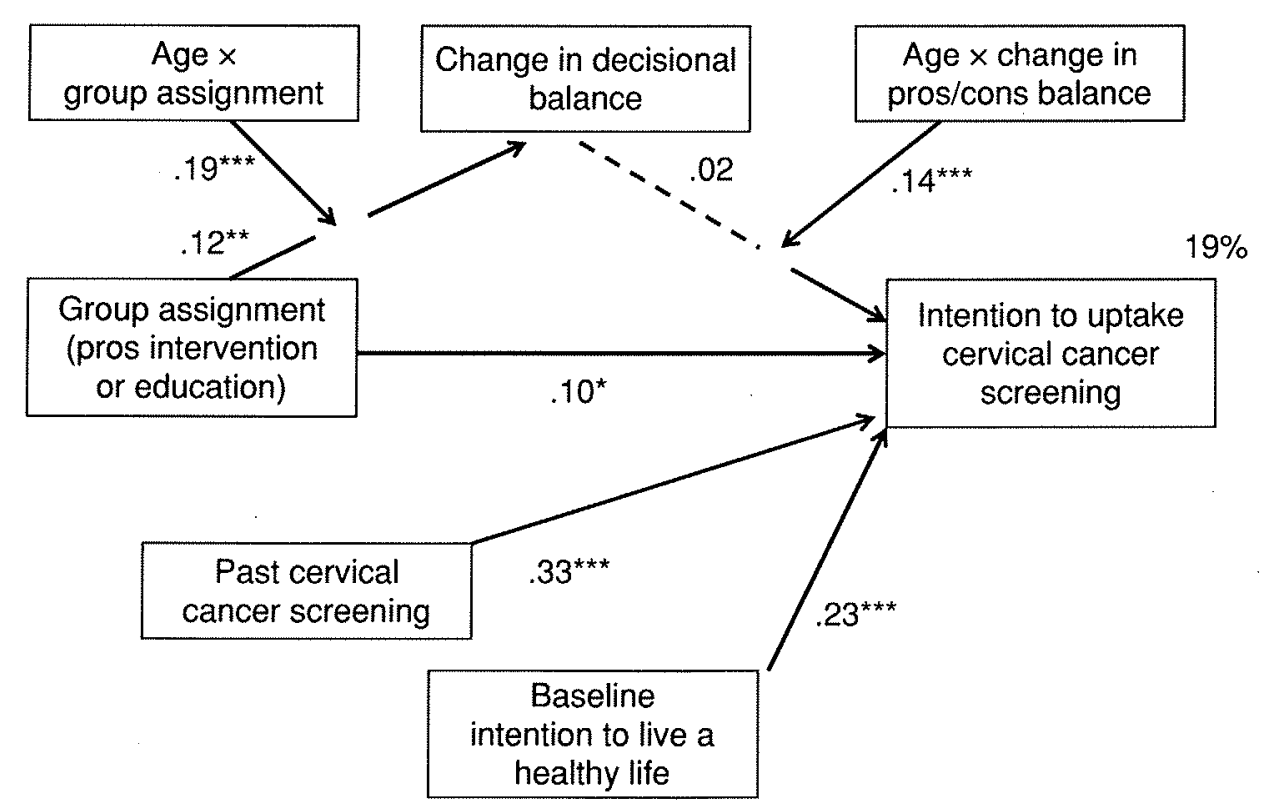

Figure 2. Moderated mediation analysis among women in preintentional stage $(N=524)$ : mediating effects of change in decisional balance are moderated by age. Note. ${ }^{*} p<.05 ;{ }^{* *} p<.01 ;{ }^{* * *} p<.001$; mediation becomes significant among women age 34.3 years or older. Solid lines represent significant relationships. 


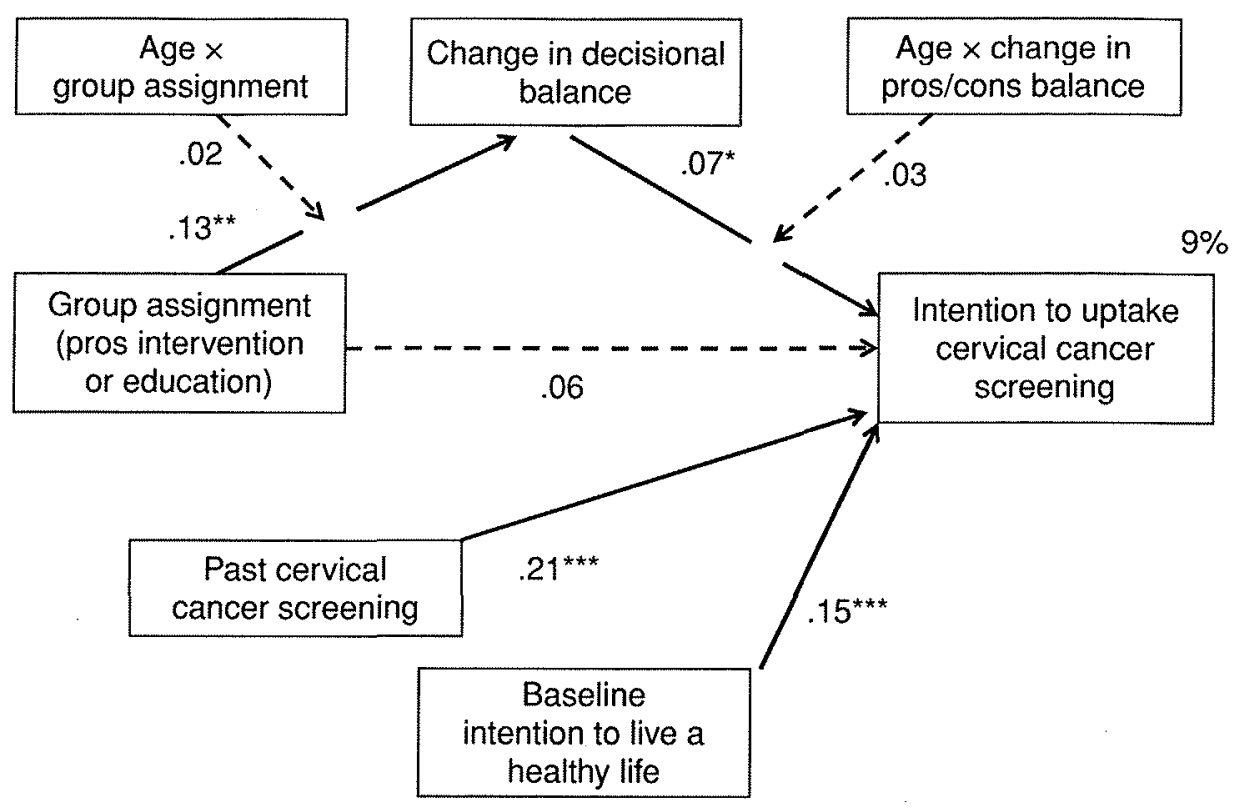

Figure 3. Moderated mediation analysis among women in intentional and actional stages $(N=9 / 2)$ : mediating effects of change in decisional balance. Note. ${ }^{*} p<.05 ;{ }^{* *} p<.01 ;{ }^{* * *} p<.001$. Solid lines represent significant relationships.

to the group assignment $(B=0.04, S E=0.02, p=.06$ ). The relationship between the decisional balance change and intention was not moderated by age, $B=0.01(S E=0.01$, ns). Finally, the two covariates had an effect on intention: baseline intention to live a healthy life, $B=0.03$ ( $S E=0.01, p<.001$ ), and past behaviour, $B=0.04$ ( $S E=0.01$, $p<.001$ ). Figure 3 yields standardized solution for the participants in intentional and actional stages.

\section{Discussion}

A brief intervention enhancing perceived advantages may affect perceived pros of cervical cancer screening and predict intention to uptake screening. The results suggest that effectiveness of this intervention depended (to some degree) on the stage of behaviour change. In particular, the direct effects on intention were observed among women in preintentional stages which can be interpreted as supporting the stage models. No direct effects were found among participants of more advanced stages. Our results corroborate findings by Lippke et al. (2004) indicating that stage-matched intervention may promote stage progression. It has to be noted that the stability of decisional balance was high, therefore the observed direct effect of the treatment should be referred to as changes in the mean level.

Although small direct effects of the intervention could be interpreted as partially supporting the concept of stages of change, the analysis of underlying mediating processes (Michie \& Abraham, 2004) provided weak support for the HAPA. An aspect that underscores the potential importance of a stage approach to some degree, however, was that the mechanisms observed in preintentional stage were different from mechanisms found for more advanced (i.e., intentional and actional) stages: in the preintentional stage (receiving the stage-matched intervention), the change in decisional balance was only identified as a mediator for women 34.3 years or older, no overall indirect effect for 
decisional balance was found. In more advanced stages, moderated mediation analysis indicated that the group assignment affected a change in decisional balance for all age groups, which in turn predicted the intention to perform cervical cancer screening. This indirect effect was small, but significant. Among women who participated in the stage-mismatched intervention, the influence of the intervention (if any) should not be explained by the mediating processes which are expected to be relevant in other stages of change (see Sutton, 2005).

Our findings add to the criticism towards stage models (Sutton, 2005; West, 2005). The regulatory mechanisms, explaining why the intervention affected the intention to uptake screening are not specific for stages. In general, the results are rather in line with the continuum models of behaviour change, which assume that shift in cognitions would always benefit intention (Schwarzer, 2008; Sutton, 2005, 2008).

Age moderated the effects of the intervention among preintentional women: among participants younger than 34.3 benefits of the intervention were limited. These results suggest that cervical cancer screening interventions should be tailored to participants' age. It is necessary to identify if other cognitions may better predict intentions to uptake screening among younger women. Future interventions should test if other strategies, such as receiving cues to actions or addressing social norms (Kahn, Goodman, Slap, Huang, \& Emmans, 2001) may result in larger effects among young participants. In line with earlier research (Byrd et al., 2004; Duffett-Leger et al., 2008; Langille \& Rigby, 2006; Tacken et al., 2007), the present study suggested that younger women are less likely to engage in cervical cancer screening, therefore young women are in particular need for the interventions promoting screening behaviours.

Our study has several limitations, which are related to its design. Stronger conclusions could be drawn if the intention and pros were measured at a longer-term follow-up rather than directly after the manipulation. This is an obvious weakness of the study and we cannot draw any conclusions regarding any longer-term effects. Stability of intention may be one of key moderators of intention-behaviour relationship (Conner \& Godin, 2007), therefore it should be accounted for in future research. Although age cohorts who are recommended to uptake cervical cancer screening according to broad guidelines were represented (18-60 years old; Hakama et al., 2008), a majority of the respondents were in their early or middle adulthood. Testing for the effects of one intervention matching only preintentional participants limits any conclusions, which cannot be generalized to interventions addressing other processes which enhance intentions.

Future studies may test the role of other social (socio-economic status), cognitive (e.g., perceived cervical cancer susceptibility, knowledge), and environmental (recommendations received form general practitioner or a specialist) moderators which may help to further explain the relationships between a pros enhancing intervention, change in decision balance, and intentions to uptake cervical cancer screening. Further investigation should test if pros enhancing interventions may evoke long-term changes in decisional balance, intention, and actual screening behaviours.

Besides its limitations, our study provides some insight into the processes explaining women's intention to uptake cervical cancer screening. First, we found that the intervention addressing pros shifted participants' decisional balance, and this change was translated into an increase of the intention to uptake screening. This was the case for middle-aged women across stages of change and among preintentional women who were 34.3-60 years old. Participating in the pros enhancing intervention, directly (stagematched) or indirectly (stage-mismatched procedure) affected women's willingness to uptake screening. 


\section{References}

Adab, P., Marshall, T., Rouse, A., Randhawa, B., Sangha, H., \& Bhangoo, N. (2003). Randomised controlled trial of the effect of evidence based information on women's willingness to participate in cervical cancer screening. Journal of Epidemiology and Community Health, 57, 589-593. doi:10.1136/jech.57.8.589

Aiken, L. S., \& West, S. G. (1991). Multiple regression: Testing and interpreting interactions. Thousand Oaks, CA: Sage.

Byrd, T. L., Peterson, S. K., Chavez, R., \& Heckert, A. (2004). Cervical cancer screening beliefs among young Hispanic women. Preventive Medicine, 38, 192-197. doi: 10.1016/ j.ypmed.2003.09.017

Conner, M., \& Godin, G. (2007). Temporal stability of intention as a moderator of intention-health behavior relationships. Psychology and Health, 22, 875-897.

De Vuyst, H., Clifford, G., Li, N., \& Franceschi, S. (2009). HPV infection in Europe. European Journal of Cancer, 45, 2632-2639. doi:10.1016/j.ejca.2009.07.019

Duffett-Leger, L. A., Letorneau, N. L., \& Croll, J. C. (2008). Cervical cancer screening practices among university women. Journal of Obstetric, Gynecologic, and Neonatal Nursing, 37, 572-581. doi:10.1111/j.1552-6909.2008.00276.x

Enders, C. K. (2001). A primer on maximum likelihood algorithms available for use with missing data. Structural Equation Modeling, 8, 128-141. doi:10.1207/S15328007SEM0801_7

Fernandez-Esquer, M. E., Espinoza, P., Torres, I., Ramirez, A. G., \& McAlister, A. L. (2003). A Su Salud: A quasi-experimental study among Mexican American women. American Journal of Health Behavior, 27, 536-545.

Franco, E. L., Schlecht, N. F., \& Saslow, D. (2003). The epidemiology of cervical cancer. Cancer, 9, 348-359. doi:10.1097/00130404-200309000-00004

Hakama, M., Coleman, M. P., Alexe, D. M., \& Auvien, A. (2008). Cancer screening: Evidence and practice in Europe 2008. European Journal of Cancer, 44, 1404-1413. doi:10.1016/j.ejca. 2008.02.013

Hogenmiller, J. R., Atwood, J. R., Lindsey, A. M., Johnson, D. R., Hertzog, M., \& Scott, J. C., Jr. (2007). Self-efficacy scale for pap smear screening participation in sheltered women. Nursing Research, 56, 369-377. doi:10.1097/01.NNR.0000299848.21935.8d

Hou, I. S., Fernandez, M. E., \& Chen, P. H. (2003). Correlates of cervical cancer screening among women in Taiwan. Health Care for Women International, 24, 384-398. doi:10.1080/ 07399330390212171

Janis, I. L., \& Mann, L. (1977). Decision making. New York: Free Press.

Kahn, J. A., Goodman, E., Slap, G. B., Huang, B., \& Emmans, S. J. (2001). Intention to return for papanicolaou smears in adolescent girls and young women. Pediatrics, 108, 333-341. doi:10.1542/peds.108.2.333

Kelaher, M., Gillespie, A. G., Allotey, P., Manderson, L., Potts, H., Sheldrake, M., \& Young, M. (1999). The transtheoretical model and cervical screening: Its application among culturally diverse communities in Queensland, Australia. Ethnicity and Health, 4, 259-276. doi:10. 1080/13557859998047

Langille, D., \& Rigby, J. (2006). Factors associated with pap testing among adolescents in northern Nova Scotia. Canadian Journal of Public Health, 97, 183-186.

Lippke, S., Ziegelmann, J. P., \& Schwarzer, R. (2004). Initiation and maintenance of physical exercise: Stage-specific effects of a planning intervention. Researcb in Sports Medicine, 12, 221-240. doi:10.1080/15438620490497567

Lippke, S., Ziegelmann, J. P., \& Schwarzer, R. (2005). Stage-specific adoption and maintenance of physical activity: Testing a three-stage model. Psychology of Sport and Exercise, 6, 585-603. doi:10.1016/j.psychsport.2004.11.002

Michie, S., \& Abraham, C. (2004). Interventions to change health behaviours: Evidence based or evidence inspired? Psychology and Health, 19, 29-49. doi:10.1080/0887044031000141199 
Preacher, K. J., \& Hayes, A. F. (2004). SPSS and SAS procedures for estimating indirect effects in simple mediation models. Bebavior Research Methods, Instruments, and Computers, 36 , 717-731.

Preacher, K. J., Rucker, D. D., \& Hayes, A. F. (2007). Addressing moderated mediation hypotheses: Theory, methods, and prescriptions. Multivariate Behavioral Research, 42, 185-227.

Rakowski, W., Ehrich, B., Dube, C. A., Pearlman, D. N., Goldstein, M. G., Rimer, B. K., ... Peterson, K. (1996). Screening mammography and the transtheoretical model: Robustness of associations using two definitions of the stages-of-adoption. Annals of Bebavioral Medicine, 18, 91-100. doi:10.1007/BF02909581

Rakowski, W., Ehrich, B., Goldstein, M. G., Rimer, B. K., Pearlman, D. N., Clark, M. A., ... Woolverton, H. (1998). Increasing mammography among women aged 40-74 by use of a stage-matched, tailored intervention. Preventive Medicine, 27, 748-756. doi:10.1006/pmed. 1998.0354

Renner, B., \& Schwarzer, R. (2005). The motivation to eat a healthy diet: How intenders and nonintenders differ in terms of risk perception, outcome expectancies, self-efficacy, and nutrition. Polish Psychological Bulletin, 36, 7-15.

Rimer, B. K., Conaway, M. R., Lyna, P. R., Glassman, B., Yarnall, K. S. H., Lipkus, I., \& Barber, L. T. (1999). The impact of tailored interventions on a community health center population. Patient Education and Counseling, 37, 125-140. doi:10.1016/S0738-3991(98)00122-0

Schuez, B., Sniehotta, F. F., Mallach, N., Wiedemann, A., \& Schwarzer, R. (2009). Prediction of stage transition in dental hygiene: Non-intenders, intenders, and actors. Health Education Research, 24, 64-75. doi:10.1093/her/cym092

Schwarzer, R. (2008). Modelling health behaviour change: How to predict and modify the adoption and maintenance of health behaviors. Applied Psychology: An International Review, 57, 1-29.

Smith, S. L., Kloss, J. D., Kniele, K., \& Anderson, S. S. (2007). A comparison of writing exercises to motivate young women to practise breast self-examinations. British Journal of Health Psychology, 12, 111-123. doi:10.1348/135910706X93637

Sutton, S. (2005). Stage theories of health behaviour. In M. Conner \& P. Norman (Eds.), Predicting health behaviour (pp. 223-275). Maidenhead: Open University Press.

Sutton, S. (2008). How does the health action process approach (HAPA) bridge the intentionbehavior gap? An examination of the model's causal structure. Applied Psychology: An International Review, 57, 66-74. doi:10.1111/j.1464-0597.2007.00326.x

Tacken, M. A. J. B., Braspenning, J. C. C., Hermens, R. P. M. G., Spreeuwenberg, P. M. M., van den Hoogen, H. J. M., de Bakker, D. H., .. . Grol, R. P. T. M. (2007). Uptake of cervical cancer screening in The Netherlands is mainly influenced by women's beliefs about screening and by the inviting organization. European Journal of Public Health, 17, 178-185. doi:10.1093/ eurpub/ckl082

Tung, W. C., Nguyen, D. H. T., \& Tran, D. N. (2008). Applying the transtheoretical model to cervical cancer screening in Vietnamese-American women. International Nursing Review, 55, 73-80. doi:10.1111/j.1466-7657.2007.00602.x

West, R. (2005). Time for a change: Putting the transtheoretical (stages of change) model to rest. Addiction, 100, 1036-1039. doi:10.1111/j.1360-0443.2004.00995.x 